\title{
The effect of slaughtering skills on the welfare of cattle during stunning with a captive bolt
}

\author{
Vladimír Večerek ${ }^{1}$, Eva Voslářová ${ }^{1}$, Josef Kameník², Zuzana Machovcová1, \\ Lenka Válková ${ }^{1}$, Martina Volfová1, Jarmila Konvalinová ${ }^{1}$
}

\begin{abstract}
University of Veterinary and Pharmaceutical Sciences Brno, Faculty of Veterinary Hygiene and Ecology, ${ }^{1}$ Department of Animal Protection and Welfare and Veterinary Public Health, ${ }^{2}$ Department of Animal Origin Food and Gastronomic Sciences, Brno, Czech Republic
\end{abstract}

Received September 29, 2020
Accepted February 24, 2021

\begin{abstract}
The performance of stunning in various abattoirs can differ. The aim of the study was to compare the stunning of cattle with a captive bolt in two abattoirs. We monitored the slaughtering skills by measuring the deviation of the location of the stunning shot hole on the skull from the ideal point and further by measuring the angle of inclination of the stunning shot on the skull from the ideal perpendicular angle. We observed the impact of different slaughter skills on the quality of stunning of animals based on the occurrence of failure to achieve motor paralysis after a stun shot. The failure to collapse occurred significantly more frequently $(P<0.05)$ in abattoir A than in abattoir B. In both abattoirs there was a higher $(P<0.05)$ number of bulls failing to collapse than in females (cows and heifers). However, the effect of slaughter skills on the occurrence of signs associated with insufficient stunning was not found. The signs occurred in abattoir A and abattoir B to the same extent. In bulls, the number of animals with signs was higher $(P<0.05)$ than in females in both abattoirs. The results show that insufficient proficiency of skills in stunning cattle with a captive bolt leads to a higher number of animals failing to collapse after a stun shot. The effect of slaughter skills on the occurrence of signs in bulls and females was not proven; however, in bulls a higher incidence of signs was demonstrated than in females.
\end{abstract}

Bull, cow, slaughter, stunning effectiveness, welfare

Slaughtering of cattle in abattoirs includes, according to the legislation, the stunning of cattle and their subsequent killing by bleeding (European Union 2009). From the welfare perspective, the stunning phase when the animal is deprived of consciousness is particularly important. Subsequent killing takes place when the animal is already unconscious, does not perceive pain and is not exposed to suffering due to environmental and human influences, the presence of other animals in the abattoir or to movement restrictions, changes in body position, and loss of coordination (Atkins on et al. 2013; Terlouw et al. 2016; Kamenik et al. 2019).

Stunning of cattle is most often performed using a device with a captive bolt, which causes sudden trauma to the skull, brain, and related blood vessels (Kamenik et al. 2019). The correct placement of the shot leads to the stunning of the animal accompanied by motor paralysis and a fall of the animal in the stunning box. In a properly stunned animal, there should be no signs associated with insufficient stunning of the animal, namely the corneal reflex, reactions to painful stimuli, the attempt to regain an upright posture, vocalization and rhythmic breathing (Gouveia et al. 2009; Atkins on et al. 2013).

Stunning quality in different abattoirs may vary and is affected by the qualifications, experience and diligence of the staff performing stunning as well as the companies' own quality assurance system, e.g. monitoring procedures with regard to stunning effectiveness (Hemsworth et al. 2011; von Wenzlawowicz et al. 2012; Atkinson et al. 2013; 
Dorfler et al. 2013). In case the stunning is not performed exactly as required, motor paralysis is not induced and the animal fails to collapse in the stunning box or shows signs associated with consciousness and perception of stress, pain, and suffering. The animal is distressed by the imperfect act of stunning, or it perceives pain and suffers. The welfare level of the slaughtered animal is thus significantly impaired (Gouveia et al. 2009) and another stun shot or even several shots are then necessary.

Also, the structure of the skull of bulls (males) and cows and heifers (females) is somewhat different (Gracey et al. 1999; Atkinson et al. 2013) and a stronger cartridge may be required. The use of less effective stunning apparatuses may result in insufficient stunning manifested by an incomplete loss of consciousness and imperfect motor paralysis and the occurrence of signs accompanying consciousness or only incomplete loss of consciousness. In case of deficiencies in stunning, differences in the response to insufficient stunning between males and females may be also apparent.

The aim of the study was to compare the stunning of cattle in two different abattoirs with staff with different levels of skills, and thereby, the impact of the skills on the occurrence of incomplete loss of consciousness in cattle accompanied by insufficient motor paralysis and a failure to collapse in the stunning box or reduced consciousness and perception of stress, pain and suffering, in order to verify that the slaughtering skills have an effect on the welfare of the slaughtered cattle. Furthermore, the aim of the study was to find out whether the slaughtering skills in stunning cattle have a different effect on the welfare of slaughtered males (bulls) and females (cows and heifers).

\section{Materials and Methods}

Animals and abattoirs

The monitoring was carried out during routine slaughtering in the two largest beef abattoirs in the Czech Republic. The stunning of cattle was monitored in each abattoir for two working days. A total of 382 heads of cattle (166 bulls, 216 cows and heifers) were slaughtered in abattoir A and 235 animals (96 bulls, 139 cows and heifers) were slaughtered in abattoir B during the monitored period. The stunning was performed in the same way at both facilities: it took place in a conventional stunning box without a mechanical head or body restrainers. When the animal's head was in a suitable position, it was shot with a Matador SS 3000 B trigger-activated captive-bolt gun (Termet, France) using calibre 6.3/12 red explosive cartridges (with a powder content of $320 \mathrm{mg}$ ) intended for all animals over $450 \mathrm{~kg}$ (bulls, young cattle, cows, heifers). The stunning was carried out by certified slaughtermen who had received relevant training as required by the Czech legislation. Two guns were available and were used alternately for each shooting to limit over-heating. The slaughter rate was approximately 40 animals per hour.

\section{Stunning quality}

Two observers were standing behind the stunning pen and collected data. During slaughtering, the number of animals was recorded where multiple stun shots had to be used to achieve motor paralysis and the required fall of the animal in the stunning box, as well as the number of animals where only one stun shot was sufficient to achieve motor paralysis and the required fall of the animal.

Furthermore, the number of animals was recorded in which reflexes/reactions occurred after stunning, being the sign of insufficient stunning of the animal: the corneal reflex, reactions to painful stimuli, attempt to regain an upright posture, vocalization and rhythmic breathing. From the values obtained, the number of animals showing signs of insufficient stunning and thereby animal welfare breach was calculated separately for each abattoir and separately for males (bulls) and females (cows and heifers).

\footnotetext{
Shot accuracy

After decapitation and skinning, the number of animals with more than one stunning shot in the skull as well as the number of animals with a single stunning shot in the skull was determined. Furthermore, the accuracy of the application of the stunning device (according to the requirements of Regulation No. 1099/2009) was evaluated, i.e. based on the deviation of the hole in the skull after the stunning shot from the ideal stunning point, and also based on the angle of the direction of the channel in the skull after the stunning shot from the ideal perpendicular direction when the stunning device is correctly applied to the skull. A circle of a radius of $3 \mathrm{~cm}$ with its centre in the ideal place for the penetration of the stunning bolt into the skull was considered to be a suitable place for placing the stunning shot at the skull. The size of the circle was determined in relation to the demonstration of adequate stunning skills, where the ideal spot on the skull cannot be accurately measured but is estimated by the
} 
person performing the stun; according to previous studies, this definition is sufficient with regard to stunning cattle. The inclination of the channel of passage of the stunning bolt through the skull perpendicular to the skull with a maximum deviation of 5 degrees was considered to be the corresponding inclination in the application of the stunning device. This deviation was determined in relation to the demonstration of adequate skills in stunning cattle, where the vertical application on the skull can be determined relatively easily and therefore a higher deviation is not required in terms of demonstration of skills. Based on the deviation of the hole in the skull from the corresponding circle of a radius of $3 \mathrm{~cm}$ and on the deviation of the inclination of the channel passage through the skull from the corresponding perpendicular inclination with a deviation of 5 degrees, the skills in stunning cattle in abattoirs A and B were compared.

\section{Statistical analysis}

The data were analysed using the statistical package Unistat v. 6.5. (Unistat Ltd., London, England). The statistical comparisons between the frequencies of the categorical variables of interest were performed with Chi-square test (with Yates correction) within the $2 \times 2$ contingency table procedure. When the frequencies in the contingency table were lower than 5, Fisher's exact test was used instead of Chi-square test (Zar 1999). A $P$ value of 0.05 in the tests was considered significant.

\section{Results}

The results of comparison between abattoirs A and B in terms of the skill level of the staff performing stunning based on the deviation of the skull hole from the ideal point with a corresponding circle of $3 \mathrm{~cm}$ radius and based on the deviation of the skull stunning passage channel from the corresponding perpendicular slope of 5 degrees are listed in Table 1 and Fig. 1. A statistical comparison showed a significant difference between abattoirs $\mathrm{A}$ and $\mathrm{B}$ in both monitored indicators. Abattoir A showed a higher number of animals with a deviation from the corresponding stunning bolt location than abattoir $\mathrm{B}(P=0.0000)$ and also a higher number of animals with a deviation from the perpendicular slope with a corresponding stunning shot deviation than abattoir B $(P=0.0434)$.

The results of monitoring the impact of different levels of skills of staff performing stunning in abattoirs $\mathrm{A}$ and $\mathrm{B}$ based on the number of animals required to use multiple stun shots to induce motor paralysis and the required fall of the animal in the stun box are listed in Table 2, separately for males and females. Comparison of abattoirs A and B in the number of inadequate cases of cattle stunning with the manifestation of insufficient paralysis and fall of the animal after a shot with a captive bolt is shown in Fig. 2.

Statistical comparison revealed a significant difference between the numbers of animals with inadequate stunning and thus insufficient paralysis and failure to collapse: in bulls between the facilities A and B $(P=0.001)$ and in females between the facilities A and B $(P=0.038)$. A significant difference was also found in abattoir A between males and females $(P=0.000)$ and in abattoir B between males and females $(P=0.004)$.

Table 2 and Fig. 2 show that slaughtering skills affect the welfare of cattle in abattoirs, where deficiencies in the stunning lead to a higher incidence of failure to achieve motor paralysis and a fall in the stunning box. In abattoir A with poorer skills in slaughtering, a significantly higher incidence of insufficient motor paralysis and non-fall of the animal after stunning was found than in abattoir B. In males, the occurrence of failure to achieve motor paralysis and non-fall of the animal was more frequent than in females in both facilities.

The results of the comparison of abattoirs A and B in the numbers of animals showing reflexes/reactions associated with insufficient stunning of the animal and in the numbers of animals showing no signs associated with insufficient stunning of the animal are listed in Table 3, separately for males and for females. Comparison of abattoirs A and B with the manifestation of the occurrence of signs associated with insufficient stunning is shown in Fig. 3.

Statistical comparison revealed no significant difference between the numbers of animals with inadequate stunning of cattle with reflexes/reactions after stunning, both for males 
Table 1. Comparison of the location and direction of the stun shot in two cattle abattoirs.

\begin{tabular}{lrrr}
\hline & \multicolumn{3}{c}{ Number of animals } \\
\cline { 2 - 4 } Abattoir A & Bulls & Cows and heifers & Total \\
\hline Total slaughtered & 166 & 216 & 382 \\
Outside the circle (above the radius of $3 \mathrm{~cm}$ ) & 54 & 76 & 130 \\
Wrong angle (deviation of $>5^{\circ}$ from the perpendicular) & 98 & 83 & 181 \\
Abattoir B & & 139 & 235 \\
\hline Total slaughtered & 96 & 14 \\
Outside the circle (above the radius of $3 \mathrm{~cm}$ ) & 14 & 53 & 28 \\
Wrong angle (deviation of $>5^{\circ}$ from the perpendicular) & 38 & & 91 \\
\hline
\end{tabular}

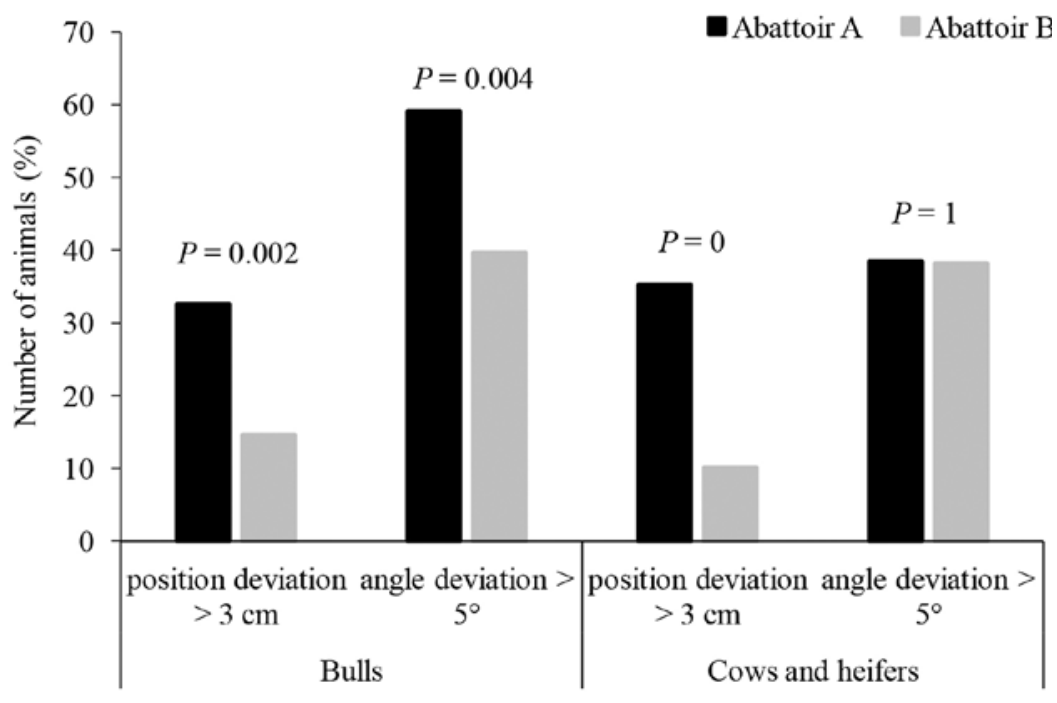

Fig. 1. Comparison of the skills of staff performing the stunning in the location and angle of inclination of the stun shot during slaughter of cattle between two abattoirs. $P=$ level of significance.

Table 2. Comparison of the effectiveness of cattle stunning in two cattle abattoirs.

\begin{tabular}{ccccc}
\hline & \multicolumn{4}{c}{ Number of animals } \\
\cline { 2 - 6 } & $\begin{array}{c}\text { More than one } \\
\text { shot fired during } \\
\text { stunning }\end{array}$ & $\begin{array}{c}\text { More than one } \\
\text { shot hole found } \\
\text { on the skull }\end{array}$ & $\begin{array}{c}\text { Insufficient } \\
\text { stunning } \\
\text { in total }\end{array}$ & $\begin{array}{c}\text { Effective } \\
\text { stunning } \\
\text { in total }\end{array}$ \\
\hline Abattoir A Bulls $(\mathrm{n}=166)$ & 54 & 24 & 54 & 112 \\
Cows and heifers $(\mathrm{n}=216)$ & 16 & 11 & 16 & 200 \\
\hline Abattoir B Bulls $(\mathrm{n}=96)$ & 12 & 12 & 12 & 84 \\
Cows and heifers $(\mathrm{n}=139)$ & 4 & 4 & 4 & 135 \\
\hline
\end{tabular}




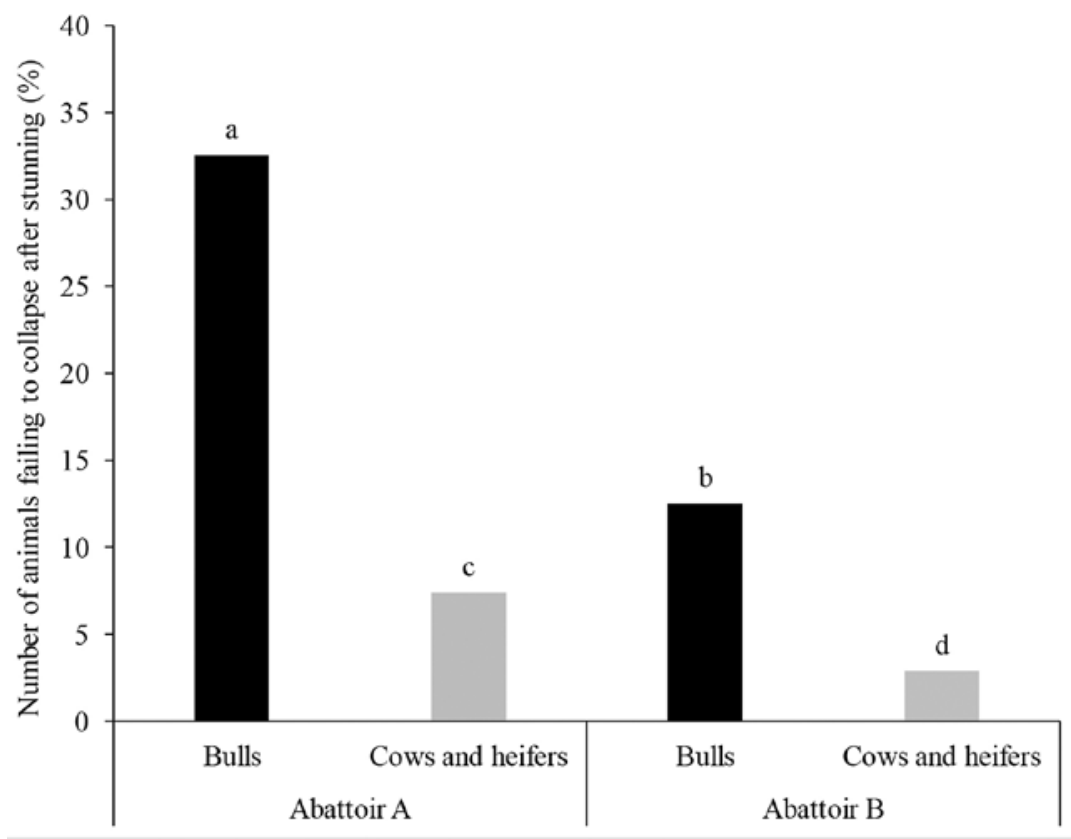

Fig. 2. Comparison of two abattoirs in the number of inadequate stunning of cattle with the manifestation of insufficient paralysis and non-fall of the animal.

${ }^{\text {a-d }}$ percentages in columns lacking a common superscript differ $(P<0.05)$

Table 3. Number of cases of inadequate cattle stunning with manifestations of signs associated with insufficient stunning in two cattle abattoirs.

\begin{tabular}{|c|c|c|c|c|}
\hline \multirow[b]{2}{*}{ Sign } & \multicolumn{2}{|c|}{$\begin{array}{c}\text { Abattoir A } \\
\text { (number of animals) }\end{array}$} & \multicolumn{2}{|c|}{$\begin{array}{c}\text { Abattoir B } \\
\text { (number of animals) }\end{array}$} \\
\hline & $\begin{array}{c}\text { Bulls } \\
(\mathrm{n}=166)\end{array}$ & $\begin{array}{l}\text { Cows and heifers } \\
\qquad(\mathrm{n}=216)\end{array}$ & $\begin{array}{c}\text { Bulls } \\
(\mathrm{n}=96)\end{array}$ & $\begin{array}{l}\text { Cows and heifers } \\
\qquad(\mathrm{n}=139)\end{array}$ \\
\hline Corneal reflex & 15 & 8 & 3 & 1 \\
\hline Response to painful stimuli & 5 & 4 & 1 & 0 \\
\hline Attempt to regain upright posture & 5 & 10 & 2 & 8 \\
\hline Vocalization & 3 & 0 & 4 & 0 \\
\hline Rhythmic breathing & 20 & 2 & 12 & 1 \\
\hline $\begin{array}{l}\text { Presence of reflexes/ } \\
\text { reactions after stunning in total }\end{array}$ & 34 & 15 & 16 & 9 \\
\hline $\begin{array}{l}\text { Absence of reflexes/ } \\
\text { reactions after stunning in total }\end{array}$ & 132 & 201 & 80 & 130 \\
\hline
\end{tabular}




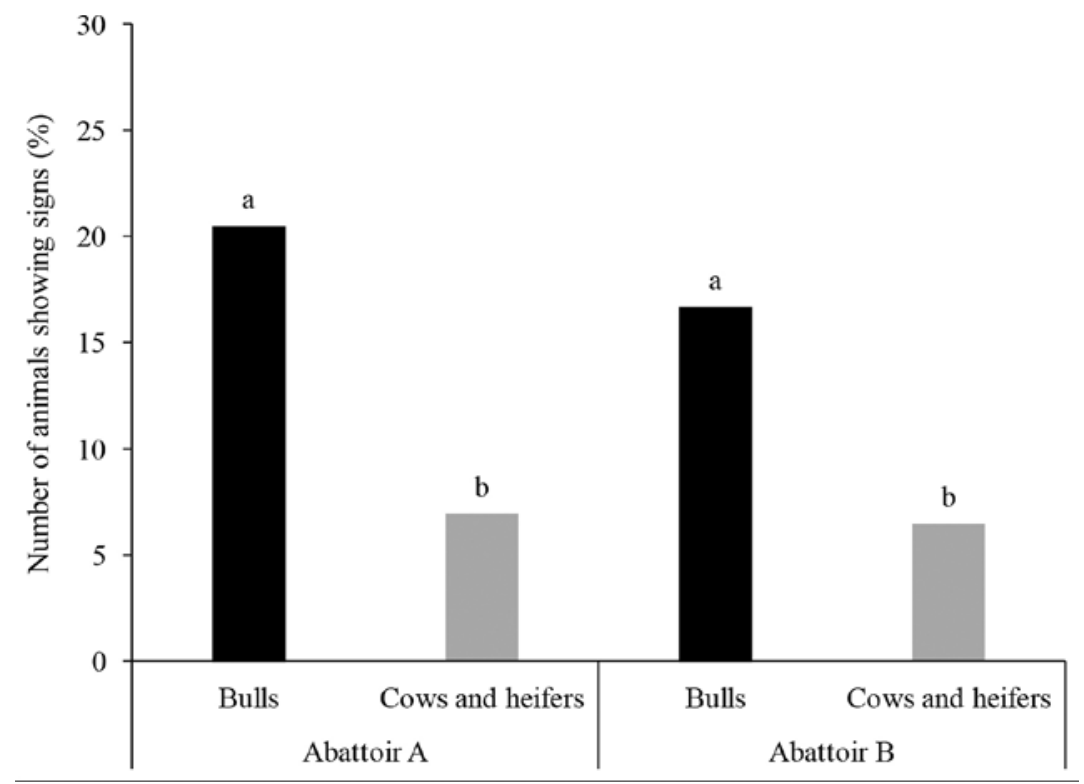

Fig. 3. Comparison of two abattoirs in the number of inadequate stunning of cattle with the manifestation of signs associated with insufficient stunning.

a,b percentages in columns lacking a common superscript differ $(P<0.05)$.

between the abattoirs A and $\mathrm{B}(P=0.553)$ and for females between the same facilities $(P=0.999)$. A significant difference was found for abattoir A between males and females $(P=0.000)$ and also for abattoir B between males and females $(P=0.023)$.

Table 3 and Fig. 3 show that the different skills of staff performing the stunning in abattoir A compared with abattoir B did not affect the occurrence of signs associated with insufficient stunning, both in males and in females. In males, the signs associated with insufficient stunning were more frequent than in females in both facilities.

\section{Discussion}

The correct position and direction of the stun shot are among the key parameters in stunning cattle with a captive bolt (European Union 2009). To achieve proper stunning, the captive bolt device must be correctly placed and directed. When evaluating the location and direction of the stunning bolt, significant differences were found in our study between the two monitored abattoirs, which demonstrates the different accuracy and care in stunning cattle in practice. Abattoir A showed a lower degree of skilfulness in slaughtering cattle compared to abattoir B, causing a presumption of worse results in the stunning of cattle. This was confirmed by the comparison of the occurrence of failure to achieve motor paralysis and non-fall of the animal in the stunning box in the two abattoirs, thereby proving the effect of slaughtering skills on the welfare of cattle at the time of stunning in an abattoir. Different degrees of care in performing the stunning have an impact on its success. While in abattoir A the motor paralysis was not induced after the first stunning shot in $18.3 \%$ of animals, in abattoir B it was only $6.8 \%$ of animals. With regard to the welfare of slaughtered animals, it is desirable to induce unconsciousness immediately after the 
first stunning shot. When properly performed, frontal captive bolt stunning can be $100 \%$ successful in adult cattle (Daly et al. 1988; Grand in 1998; Gregory and Shaw 2000). This was not achieved in any of the facilities monitored; moreover, in abattoir A, significantly more animals were exposed to stress, pain, and suffering before an effective stun. Some previous studies also point to variability in efficiency of penetrative bolt stunning in cattle in commercial abattoirs. For instance, according to Gouveia et al. (2009), in an abattoir in Portugal the overall efficiency of captive bolt stunning was only $68.2 \%$. A study by Atkinson et al. (2013) points out a Swedish abattoir with $12.5 \%$ of cattle improperly stunned. Von Wenzlawowicz et al. (2012) found significant differences between selected abattoirs in Austria, Germany, and Switzerland with the insufficiency of stunning in 9.2\% of slaughtered cattle. Although the procedure of stunning by a device with a captive bolt is uniformly prescribed by legislation (European Union 2009), in practice the compliance with this procedure depends on a number of factors. For example, Vecerek et al. (2020a) demonstrated the importance of accuracy of the stun shot as they found the failure to induce motor paralysis in cattle increasing with the deviation in the stun shot position. Placing the stunning device in the correct position depends on the level of experience of the person performing the stun. Dorfler et al. (2013) point out the different efficiency of stunning by an experienced slaughterman or by a substitute. Atkin son et al. (2013) also found the most frequent occurrence of imperfect stunning in the case of a slaughterman who had worked in the abattoir for a short time compared to other four employees with an experience of three or more years. The lowest percentage of improper stun shots was in the case of a shooter with 15 years of experience. It is obvious that the staff engaged in the stunning and slaughtering of animals play a crucial role in the welfare of animals in abattoirs. The abattoir management should ensure that the employees of the abattoir are competent and carry out their tasks according to the principles of animal welfare. Maintaining a high standard of welfare requires constant attention and vigilance of the management. As a consequence, abattoirs with competent management constantly working on improving the procedures report a high level of animal welfare (Grandin 1988, 1994).

Slaughtering skills have an effect on the welfare of slaughtered cattle. However, the results showed that the slaughterman's skills are crucial, especially in stunning bulls, which requires repeating the stun shot to achieve motor paralysis more often than in cows and heifers. While in abattoir A more than one stun shot was used in $32.5 \%$ of bulls, only $7.4 \%$ of females did not fall after the first shot. In abattoir B, more than one stunning shot was used in $12.5 \%$ of males and in $2.9 \%$ of females. The structure of the skull of bulls and cows is to some extent different, which may therefore result in the occurrence of insufficient stunning manifested by an incomplete loss of consciousness with insufficient motor paralysis. Since bulls generally have a higher weight and thus a thicker bone mass on the forehead in comparison to females, they also show a higher resistance to the kinetic energy of the stun bolt (Atkinson et al. 2013). Similarly, Gracey et al. (1999) pointed out that as a result of a larger frontal bone, the brain of some bulls may be beyond the standard reach of the bolt. The deficiencies in stunning can lead to a failure to collapse after a stun shot, especially in bulls. The abattoir operators have to ensure that standard operating procedures are implemented in order that the animal welfare rules are properly understood and applied.

With regard to the occurrence of signs associated with insufficient stunning, the effect of slaughtering skills on the occurrence of lower welfare standards at the time of stunning in the abattoir was not found. The varying diligence of stunning did not affect the incidence of an incomplete loss of consciousness in cattle accompanied by signs associated with insufficient stunning. This is consistent with the findings of studies reporting that the occurrence of reflexes/reactions following the stunning of cattle with a captive bolt is dependent not only on the accuracy of the shot (Atkinson et al. 2013; Vecerek et al. 
2020b). However, in both abattoirs, the signs associated with insufficient stunning were more common in bulls than in cows and heifers. Similarly, Vecerek et al. (2020b) found a difference in the occurrence of reflexes and reactions resulting from the difference in the skulls of bulls and cows or in differing sensitivity of bulls and cows to stunning with a captive bolt. Atkinson et al. (2013) also found that bulls showed symptoms rated at the highest level for inferior stun quality three times more frequently than other cattle.

In conclusion, the results show that there are differences in the skills of stunning cattle in different abattoirs, although the legislative requirements, prescribed procedures, methods used and the training of the stunning operators are the same. The differences in stunning skills between the monitored abattoirs A and B led to a differing number of animals in which the motor paralysis was not achieved immediately after the stunning shot and the process had to be repeated. These animals were exposed to pain and stress before being successfully stunned and losing consciousness and sensibility. The stunning skills thus significantly affect the welfare of cattle slaughtered in abattoirs. Therefore, it is necessary to ensure precise compliance with the prescribed procedures in abattoir operations, continuous control of the correct execution of stunning and motivation of workers. Conversely, the observed differences in stunning skills between abattoirs A and B did not lead to differences in the incidence of signs associated with insufficient stunning, both in bulls and in cows and heifers. The occurrence of these signs is affected not solely by the location and inclination of the stunning device on the skull.

\section{References}

Atkinson S, Velarde A, Algers B 2013: Assessment of stun quality at commercial slaughter in cattle shot with captive bolt. Anim Welfare 22: 473-481

Daly CC, Kallweit E, Ellendorf F 1988: Cortical function in cattle during slaughter: Conventional captive bolt stunning followed by exsanguination compared with shechita slaughter. Vet Rec 122: 325-329

Dorfler K, Troeger K, Lautenschlager R, Schonekeß HC, Jager F, Lucker E 2013: Kinetische Energie und Effektivität verschiedener Bolzenschuss-Betäubungsverfahren. Fleischwirtschaft 93: 91-96

European Union 2009: Council Regulation (EC) No 1099/2009 of 24 September 2009 on the protection of animals at the time of killing. Off J Eur Union L303: 1-30

Gouveia KG, Ferreira PG, Roque de Costa JC, Vaz-Pires P, Martins da Costa P 2009: Assessment of the efficiency captive-bolt stunning in cattle and feasibility of associated behavioural signs. Anim Welfare 18: 171-175

Gracey J, Collins DS, Huey R 1999: Humane slaughter. In: Gracey J, Collins DS, Huey R (Eds.): Meat hygiene. WB Saunders Company Ltd., London, pp. 197-222

Grandin T 1988: Behavior of slaughter plant and auction employees towards animals. Anthrozoos 1: 205-213

Grandin T 1994: Farm animal welfare during handling, transport, and slaughter. J Am Vet Med A 204: $372-377$

Grandin T 1998: Objective scoring of animal handling and stunning practices at slaughter plants. J Am Vet Med A 212: 36-39

Gregory NG, Shaw F 2000: Penetrating captive bolt stunning and exsanguination of cattle in abattoirs. J Appl Anim Welf Sci 3: 215-230

Hemsworth PH, Rice M, Karlen MG, Calleja L, Barnett JL, Nash J, Coleman GJ 2011: Human-animal interactions at abattoirs: Relationships between handling and animal stress in sheep and cattle. Appl Anim Behav Sci 135: 24-33

Kamenik J, Paral V, Pyszko M, Voslarova E 2019: Cattle stunning with a penetrative captive bolt device: A review. Anim Sci J 90: 307-316

Terlouw C, Bourguet C, Deiss V 2016: Consciousness, unconsciousness and death in the context of slaughter. Part II. Evaluation methods. Meat Sci 118: 147-156

Vecerek V, Kamenik J, Voslarova E, Volfova M, Machovcova Z, Konvalinova J, Vecerkova L 2020a: The impact of deviation of the stun shot from the ideal point on motor paralysis in cattle. Animals 10: 280

Vecerek V, Kamenik J, Voslarova E, Vecerkova L, Machovcova Z, Volfova M, Konvalinova J 2020b: The occurrence of reflexes and reactions in cattle following stunning with a captive bolt at the slaughterhouse. Anim Sci J 91: e13373

Von Wenzlawowicz M, von Holleben K, Eser E 2012: Identifying reasons for stun failures in slaughterhouses for cattle and pigs: a field study. Anim Welfare 21: 51-60

Zar JH 1999: Biostatistical Analysis. $4^{\text {th }}$ edn. Prentice-Hall, Inc., New Jersey, 663 p. 